\title{
Incidence and Effect of Intrathecal Fentanyl Use in Spinal Anesthesia for Cesarean Deliveries in the Community Setting: A Single-Center Observational Retrospective Study
}

\author{
Autumn Brewer, BS, ${ }^{1}$ Sarah Joseph, BS, ${ }^{1}$ Kendall Hammonds, MS, ${ }^{2}$ Michael P. Hofkamp, MD $^{3}$ \\ ${ }^{1}$ Texas A\&M University Health Science Center College of Medicine, Temple, TX ${ }^{2}$ Office of Biostatistics, Baylor Scott \& White Research \\ Institute, Temple, TX ${ }^{3}$ Department of Anesthesiology, Baylor Scott \& White Medical Center - Temple, Temple, TX
}

Background: The addition of intrathecal fentanyl to spinal anesthesia for cesarean delivery has been shown to be beneficial, but its rate of utilization in the community setting is unknown. The primary aim of our study was to determine the rate of intrathecal fentanyl use for cesarean deliveries with spinal anesthesia in a community hospital, and our secondary aim was to determine its effect on anesthetic outcomes.

Methods: Patients who underwent cesarean delivery from June 1, 2017 to November 30, 2019 with spinal anesthesia as the initial anesthetic technique were included.

Results: Seven hundred sixty-one cesarean deliveries met inclusion criteria, and 161 (21.2\%) patients received intrathecal fentanyl in their spinal anesthetic for cesarean delivery. A multivariate model that controlled for patient weight and time from spinal placement to procedure end showed that patients who received intrathecal fentanyl were less likely to have conversion to general anesthesia or administration of systemic anesthetic adjuncts compared to patients who did not receive intrathecal fentanyl (odds ratio $2.889,95 \% \mathrm{Cl} 1.552-5.903 ; P=0.0017)$.

Conclusion: Only 1 in 5 patients received intrathecal fentanyl for cesarean deliveries performed under spinal anesthesia in a community hospital despite known benefits. Patients who did not receive intrathecal fentanyl had increased odds of conversion to general anesthesia or administration of systemic anesthetic adjunct administration, a finding consistent with previous studies. The addition of intrathecal fentanyl to spinal anesthesia for cesarean delivery should be strongly considered in the community setting.

Keywords: Analgesia, anesthesia-conduction, anesthesia-obstetrical, cesarean section

Address correspondence to Michael Hofkamp, MD, Department of Anesthesiology, Baylor Scott \& White Medical Center - Temple, 2401 S 31st St., Temple, TX 76508. Tel: (254) 724-3370. Email: rfums2004@hotmail.com

\section{INTRODUCTION}

Anesthesia for cesarean delivery is commonly performed with spinal anesthesia that consists of a local anesthetic such as bupivacaine, a short-acting opioid such as fentanyl, and a long-acting opioid such as morphine. ${ }^{1}$ Intrathecal fentanyl has been shown to be beneficial for intraoperative pain associated with cesarean delivery, ${ }^{2}$ while intrathecal morphine has an important effect on postoperative pain. ${ }^{3}$

Conversion to general anesthesia signifies failure of spinal anesthesia for cesarean delivery, while the administration of systemic anesthetic adjunct medication such as intravenous fentanyl or inhaled nitrous oxide is suggestive of inadequate analgesia. Clevenger and colleagues found that $13.9 \%$ of patients who had spinal anesthesia as their primary anes- thetic technique for cesarean delivery required the use of systemic anesthetic adjuncts. ${ }^{4}$

The prevalence of intrathecal fentanyl administration in spinal anesthesia for cesarean delivery in the community setting is unclear. The primary aim of our study was to determine the rate of intrathecal fentanyl use in spinal anesthesia for cesarean delivery in a community setting. The secondary aim was to determine if a difference in either conversion to general anesthesia or administration of systemic anesthetic adjunct administration was found between patients who did and did not receive intrathecal fentanyl for cesarean delivery.

\section{METHODS}

After approval by the Baylor Scott \& White Research Institute Institutional Research Board, the electronic medical 
record system (Epic Systems Inc) was used to search for cesarean deliveries performed at Baylor Scott \& White Medical Center - College Station from June 1, 2017 to November 30,2019 . Patients were included if they received spinal anesthesia as the initial anesthetic technique for cesarean delivery and were excluded if they received labor epidural analgesia. Study data were collected and managed using REDCap electronic data capture tools hosted at Baylor Scott \& White Research Institute. Demographic data including age, height, weight, body mass index, gravidity, parity, history of previous cesarean delivery, and gestational age were collected. The principal investigator examined each operative note and categorized the obstetric indication of cesarean deliveries into elective, urgent, and fetal heart rate abnormalities based on whether the cesarean delivery was scheduled, unscheduled, or had fetal heart rate abnormalities documented. The attending anesthesiologist of record was recorded by assigning the letter $A$ to the first attending anesthesiologist who appeared in the study and the letter B to the second, with the process repeated through the letter J. Date and time of spinal placement for cesarean delivery and the end of the procedure were collected. Additional data collected were the neuraxial anesthetic kit used, whether the operator performing the neuraxial anesthetic was a physician anesthesiologist or certified registered nurse anesthetist, dose of hyperbaric bupivacaine $0.75 \%$, dose of intrathecal preservative-free morphine, dose of intrathecal fentanyl, whether the patient required conversion to general anesthesia, and whether the patient received anesthetic adjuncts during the cesarean delivery (intravenous fentanyl, morphine, ketamine, propofol, midazolam, inhaled nitrous oxide, and sevoflurane).

Statistical analysis was performed using SAS statistical software, version 9.4 (SAS Institute Inc). Descriptive statistics are used to describe characteristics of the sample. Frequencies and percentages are used to describe categorical variables, and medians with interquartile ranges are used to describe continuous variables. Chi-square test, Fisher exact test, or Monte Carlo estimated Fisher test was used for categorical variables, and Wilcoxon rank sum test was used to test for continuous variables to examine associations in bivariate comparisons. Multivariate logistic regression was used to examine the effect of intrathecal fentanyl use on either conversion to general anesthesia or administration of systemic anesthetic adjunct administration while controlling for selected clinically and statistically significant variables. Variables with a bivariate $P$ value $<0.10$ were considered for inclusion in the final model. All potential covariates were then included in a logistic regression and removed one at a time until only clinically significant or those with a $P$ value $<0.10$ variables remained. Statistical significance was determined at a level of 0.05 .

\section{RESULTS}

Seven hundred sixty-one cesarean deliveries met inclusion criteria for the study. Although some anesthesiologists and certified registered nurse anesthetists used a combined spinal epidural neuraxial kit to obtain spinal anesthesia, no epidural catheters were inserted into the epidural space. Six hundred patients did not receive intrathecal fentanyl, and 161 patients did: 4,153 , and 3 patients received $5 \mu \mathrm{g}, 10 \mu \mathrm{g}$, and $20 \mu \mathrm{g}$ of intrathecal fentanyl, respectively, and 1 patient received a dose of $100 \mu \mathrm{g}$ in error. Demographic and clinical data for patients who did and did not receive intrathecal fentanyl are included in the Table. Nine patients had conversion to general anesthesia prior to skin incision, and 2 patients had conversion to general anesthesia after delivery of the baby. Of the 11 patients who required general anesthesia, 1 had a failed block with a documented T10 dermatomal level, 9 had a failed block without documentation of dermatomal level, and 1 patient did not have a reason documented for conversion to general anesthesia.

Multivariate analysis designed to predict the odds of either conversion to general anesthesia or administration of systemic anesthetic adjunct medication between patients who did and did not receive intrathecal fentanyl is presented in the Figure. One hundred fifty-one patients either had conversion to general anesthesia or administration of systemic adjunct medication, and 610 patients did not. Fiftyeight physician anesthesiologists and 93 certified registered nurse anesthetists were the documented proceduralists who performed the spinal anesthesia for patients who either had conversion to general anesthesia or administration of systemic anesthetic adjunct medication compared to 251 physician anesthesiologists and 359 certified registered nurse anesthetists who were the documented proceduralists for patients who did not have either conversion to general anesthesia or administration of systemic anesthetic adjunct medication, a finding that was not statistically significant $(P=0.540)$. Attending anesthesiologists C, F, and $\mathrm{G}$ used intrathecal fentanyl $59.1 \%, 30.8 \%$, and $37.1 \%$ of the time, respectively, and collectively had conversion to general anesthesia or administration of systemic adjuncts at a rate of $14.0 \%$ compared to a rate of $22.0 \%$ for their colleagues, a difference that was statistically significant $(P=0.004)$.

\section{DISCUSSION}

We found that only $21.2 \%$ of patients who underwent cesarean delivery with spinal anesthesia as the initial anesthetic technique received intrathecal fentanyl, and we attributed this finding to variances in the individual practice of the attending anesthesiologists of record. Only 10 attending anesthesiologists were included in our study, and we did not report any demographic data such as years of experience that could compromise their anonymity. We are unaware of any cost containment issues associated with restricting the use of intrathecal fentanyl for cesarean delivery. Our multivariate model showed that patients who did not receive intrathecal fentanyl had an approximate $189 \%$ increase in odds of either conversion to general anesthesia or administration of systemic anesthetic adjuncts.

The addition of intrathecal fentanyl to a spinal anesthetic has been shown to lower the dose of subarachnoid hyperbaric bupivacaine needed to provide adequate anesthesia for cesarean delivery. Choi et al demonstrated that patients who received $8 \mathrm{mg}, 10 \mathrm{mg}$, and $12 \mathrm{mg}$ of intrathecal bupivacaine reported intraoperative pain at rates of $35 \%$, $20 \%$, and $0 \%$, respectively, while patients who received the same doses of intrathecal bupivacaine with the addition of intrathecal fentanyl $10 \mu \mathrm{g}$ reported no intraoperative pain. ${ }^{2} \mathrm{~A}$ 2020 meta-analysis that included 14 randomized controlled trials with 694 patients undergoing cesarean delivery with spinal anesthesia showed that 17 of 370 patients $(4.6 \%)$ 
Table. Demographic and Clinical Data by Group

\begin{tabular}{|c|c|c|c|}
\hline Variable & $\begin{array}{l}\text { Received Intrathecal } \\
\text { Fentanyl, } \\
n=161\end{array}$ & $\begin{array}{c}\text { Did Not Receive } \\
\text { Intrathecal Fentanyl, } \\
n=600\end{array}$ & $P$ Value \\
\hline Age, years & $31(26-34)$ & $30(26-34)$ & 0.387 \\
\hline \multirow[t]{2}{*}{ Height, $\mathrm{cm}$} & $165.1(160-167.6)$ & $162.6(157.5-167.6)$ & 0.059 \\
\hline & $\mathrm{n}=111$ & $\mathrm{n}=452$ & \\
\hline \multirow[t]{2}{*}{ Weight, kg } & $94.3(77.4-112.4)$ & $88.9(75.2-104.0)$ & 0.031 \\
\hline & $n=119$ & $n=493$ & \\
\hline \multirow[t]{2}{*}{ Body mass index, $\mathrm{kg} / \mathrm{m}^{2}$} & $35.0(30.1-41.1)$ & $33.3(28.9-38.9)$ & 0.078 \\
\hline & $\mathrm{n}=106$ & $\mathrm{n}=452$ & \\
\hline Gravidity & $3(2-3)$ & $3(2-4)$ & 0.517 \\
\hline Parity & $1(1-2)$ & $1(1-2)$ & 0.690 \\
\hline Gestational age, weeks & $38.7(37.7-39.1)$ & $38.9(37.6-39.1)$ & 0.945 \\
\hline \multirow[t]{2}{*}{ History of cesarean delivery, n (\%) } & Yes: 114 (70.8) & Yes: 431 (71.8) & 0.798 \\
\hline & No: 47 (29.2) & No: 169 (28.2) & \\
\hline \multirow[t]{2}{*}{ History of depression, n (\%) } & Yes: 17 (10.6) & Yes: 66 (11.0) & 0.873 \\
\hline & No: $144(89.4)$ & No: 534 (89.0) & \\
\hline \multirow[t]{2}{*}{ History of anxiety, n (\%) } & Yes: $16(9.9)$ & Yes: 72 (12.0) & 0.468 \\
\hline & No: $145(90.1)$ & No: $528(88.0)$ & \\
\hline Indication for cesarean delivery, n (\%) & & & 0.520 \\
\hline Elective & $114(70.8)$ & $417(69.5)$ & \\
\hline Urgent & $42(26.1)$ & $172(28.7)$ & \\
\hline Fetal heart rate abnormalities & $5(3.1)$ & $11(1.8)$ & \\
\hline Attending anesthesiologist of record, $\mathrm{n}(\%)$ & & & $<0.001^{\mathrm{a}}$ \\
\hline A & $4(2.5)$ & $113(18.8)$ & \\
\hline B & $1(0.6)$ & $91(15.2)$ & \\
\hline $\mathrm{C}$ & $55(34.2)$ & $38(6.3)$ & \\
\hline $\mathrm{D}$ & $11(6.8)$ & $101(16.8)$ & \\
\hline $\mathrm{E}$ & $14(8.7)$ & $100(16.7)$ & \\
\hline $\mathrm{F}$ & $36(22.4)$ & $81(13.5)$ & \\
\hline G & $39(24.2)$ & $66(11.0)$ & \\
\hline $\mathrm{H}$ & 0 & $4(0.7)$ & \\
\hline 1 & $1(0.6)$ & $5(0.8)$ & \\
\hline J & 0 & $1(0.2)$ & \\
\hline \multirow[t]{2}{*}{ Operator who performed neuraxial anesthetic, n (\%) } & MD: 77 (47.8) & MD: 232 (38.7) & 0.036 \\
\hline & CRNA: 84 (52.2) & CRNA: 368 (61.3) & \\
\hline \multirow[t]{2}{*}{ Neuraxial kit used for spinal anesthesia, n (\%) } & SSS: $152(94.4)$ & SSS: 568 (94.7) & 0.898 \\
\hline & CSE: 9 (5.6) & CSE: 32 (5.3) & \\
\hline Intrathecal bupivacaine dose, $\mathrm{mg}$ & $12.0(11.3-12.0)$ & $12.0(11.3-12.0)$ & 0.430 \\
\hline Received intrathecal morphine, $\mathrm{n}(\%)$ & $155(96.3)$ & $591(98.5)$ & 0.071 \\
\hline Intrathecal morphine dose, mg & $0.2(0.2-0.2)$ & $0.2(0.2-0.2)$ & $<0.001$ \\
\hline Time from spinal to surgery end, $\min$ & $45(39-50)$ & $43(38-49)$ & 0.055 \\
\hline Conversion to general anesthesia, $\mathrm{n}(\%)$ & $2(1.2)$ & $9(1.5)$ & 1 \\
\hline $\begin{array}{l}\text { Conversion to general anesthesia or anesthetic } \\
\text { adjunct administration, } \mathrm{n}(\%)\end{array}$ & $13(8.1)$ & $138(23.0)$ & $<0.001$ \\
\hline
\end{tabular}

${ }^{a}$ Monte Carlo analysis performed to determine Fisher exact test.

Notes: Data are presented as medians (interquartile ranges) unless otherwise noted.

CRNA, certified registered nurse anesthetist; CSE, combined spinal epidural; MD, physician anesthesiologist; SSS, single shot spinal. 


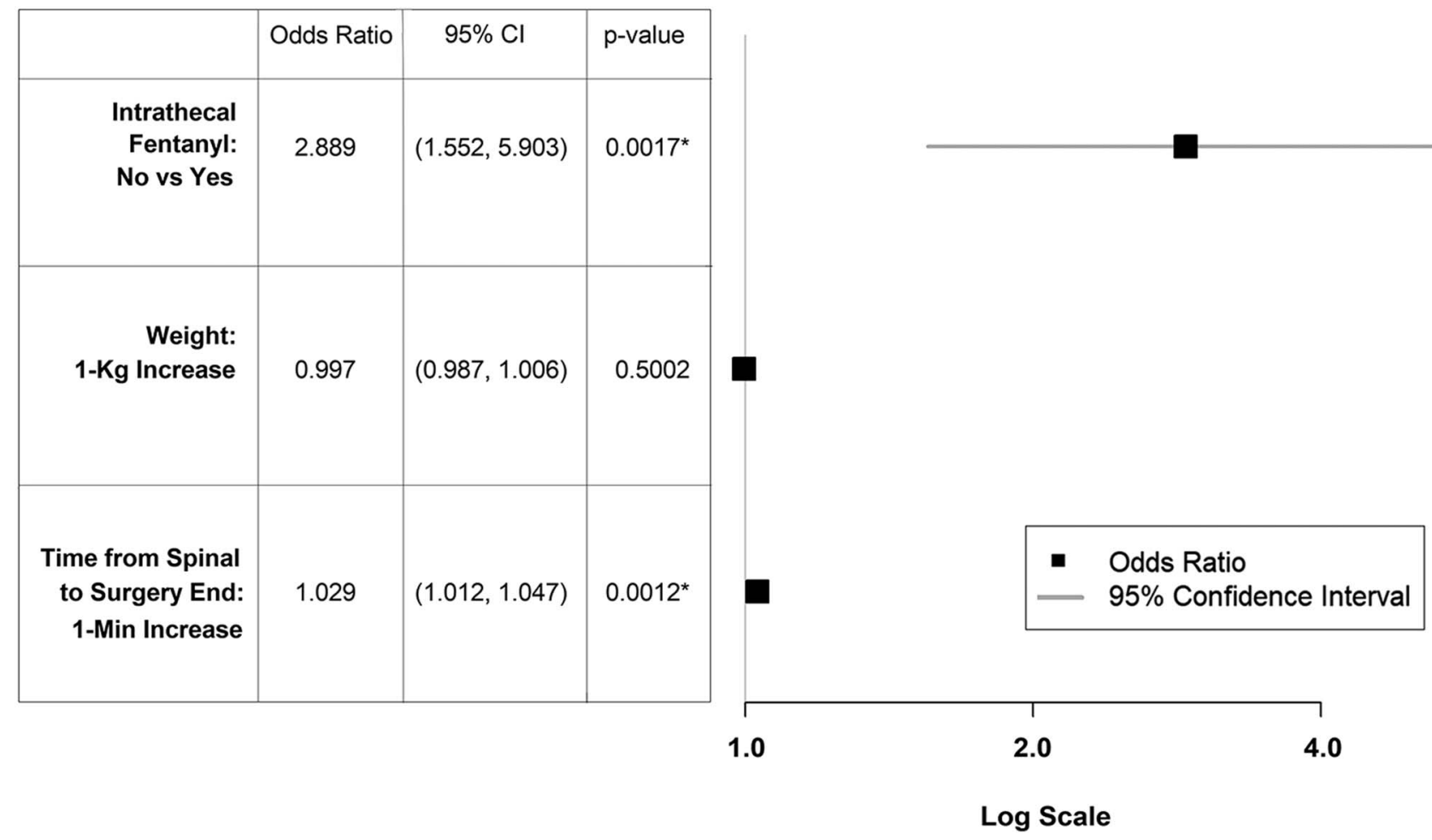

Figure. Multivariate model and forest plot show that subjects who did not receive intrathecal fentanyl had an approximate $189 \%$ increase in odds of either conversion to general anesthesia or administration of systemic anesthetic adjuncts. The area under the receiver operating characteristic curve for this model was $\mathbf{0 . 6 4 5}$. Asterisks denote significance.

who received intrathecal fentanyl with bupivacaine required systemic anesthetic adjunct medication compared to 96 of 324 (29.6\%) patients who received only bupivacaine, a result that was statistically significant $(P<0.001) .^{5}$

Increased pain is a potential consequence of using lower doses of intrathecal bupivacaine for cesarean deliveries performed with spinal anesthesia. A 2011 meta-analysis showed a higher risk for systemic anesthetic adjunct supplementation in patients who received $<8 \mathrm{mg}$ of subarachnoid bupivacaine for cesarean delivery with spinal anesthesia compared to patients who received $>8 \mathrm{mg} .{ }^{6}$ Studies published in 2004 and 2017 showed the ED95 (effective) dose of hyperbaric bupivacaine for cesarean delivery to be $11.2 \mathrm{mg}$ and $12.6 \mathrm{mg}$, respectively. ${ }^{7,8}$ In our study, patients who did and did not receive intrathecal fentanyl had identical median doses of bupivacaine of $12.0 \mathrm{mg}$.

The effect of height and weight on the dose of intrathecal bupivacaine for cesarean delivery is controversial. Hogan and colleagues demonstrated that patients with increased abdominal pressure had lower volumes of cerebrospinal fluid, ${ }^{9}$ and this finding suggests that lower doses of intrathecal bupivacaine would be needed to provide spinal anesthesia for obese patients. However, Carvalho and colleagues found that patients with a body mass index $>40 \mathrm{~kg} / \mathrm{m}^{2}$ required a similar dose of intrathecal bupivacaine for cesarean delivery compared to nonobese patients. ${ }^{10}$ In our study, patients who received intrathecal fentanyl had a higher weight compared to patients who did not receive intrathecal fentanyl, but weight was not a predictor of either conversion to general anesthesia or administration of sys- temic anesthetic adjunct administration in our multivariate model.

The relationship between previous cesarean delivery and intraoperative cesarean delivery pain is unclear. Studies have demonstrated that patients with a history of cesarean delivery tend to have more adhesions and a longer operative time, ${ }^{11,12}$ but a study from 2019 showed that patients undergoing primary cesarean delivery had increased odds of incisional pain compared to patients undergoing repeat cesarean delivery. ${ }^{13}$ In our study, we found no difference in history of cesarean delivery between patients who received intrathecal fentanyl and those who did not.

Our study has several limitations. Height and weight data for patients were incomplete. The dermatomal level from spinal anesthesia was not routinely documented. We found that patients who received intrathecal fentanyl had a higher weight compared to patients who did not receive intrathecal fentanyl, and we used this variable in our multivariate analysis. The time from spinal placement to procedure end was close to meeting statistical significance, and we included this variable in our multivariate model. We found that patients who did not receive intrathecal fentanyl had a statistically significant higher percentage of certified registered nurse anesthetists who performed the neuraxial procedure compared to patients who received intrathecal fentanyl. We examined patients who received either conversion to general anesthesia or administration of systemic anesthetic adjunct medication and found no difference in the percentage of physician anesthesiologists or certified registered nurse anesthetists performing the neuraxial procedure. 
Therefore, we attribute our finding that nurse anesthetists were statistically more likely to be the operator for a spinal anesthetic that did not use intrathecal fentanyl vs a spinal anesthetic using intrathecal fentanyl to type I error.

\section{CONCLUSION}

Intrathecal fentanyl should be strongly considered for use in spinal anesthesia for cesarean delivery in a community setting because of its ability to lower the risk of systemic anesthetic adjunct medication administration and conversion to general anesthesia.

\section{ACKNOWLEDGMENTS}

This study was departmentally funded. An abstract based on data from this study was accepted for presentation at the 2020 Society for Obstetric Anesthesia and Perinatology Annual Meeting, but that meeting was subsequently canceled because of the COVID-19 pandemic. The authors have no other financial or proprietary interest in the subject matter of this article.

\section{REFERENCES}

1. Practice guidelines for obstetric anesthesia: an updated report by the American Society of Anesthesiologists Task Force on Obstetric Anesthesia and the Society for Obstetric Anesthesia and Perinatology. Anesthesiology. 2016;124(2):270-300. doi: 10.1097/ALN.0000000000000935

2. Choi DH, Ahn HJ, Kim MH. Bupivacaine-sparing effect of fentanyl in spinal anesthesia for cesarean delivery. Reg Anesth Pain Med. 2000;25(3):240-245.

3. Sultan P, Halpern SH, Pushpanathan E, Patel S, Carvalho B. The effect of intrathecal morphine dose on outcomes after elective cesarean delivery: a meta-analysis. Anesth Analg. 2016;123(1):154-64. doi: 10.1213/ANE.0000000000001255

4. Clevenger K, Maresh B, Graham H, Hammonds K, Hofkamp MP. The use of adjunct anesthetic medication with regional anesthesia and rates of general anesthesia for 1867 cesarean deliveries from 2014 to 2018 in a community hospital. Proc (Bayl Univ Med Cent). 2020;33(4):536-540.
5. Uppal V, Retter S, Casey M, Sancheti S, Matheson K, McKeen DM. Efficacy of intrathecal fentanyl for cesarean delivery: a systematic review and meta-analysis of randomized controlled trials with trial sequential analysis. Anesth Analg. 2020;130(1):111-125. doi: 10.1213/ANE.0000000000003975

6. Arzola C, Wieczorek PM. Efficacy of low-dose bupivacaine in spinal anaesthesia for caesarean delivery: systematic review and meta-analysis. Br J Anaesth. 2011;107(3):308-318. doi: 10.1093/bja/aer200

7. Ginosar Y, Mirikatani E, Drover DR, Cohen SE, Riley ET. ED50 and ED95 of intrathecal hyperbaric bupivacaine coadministered with opioids for cesarean delivery. Anesthesiology. 2004;100(3):676-682. doi: 10.1097/00000542-200403000-00031. Erratum in Anesthesiology. 2005;102(2):489.

8. Onishi E, Murakami M, Hashimoto K, Kaneko M. Optimal intrathecal hyperbaric bupivacaine dose with opioids for cesarean delivery: a prospective double-blinded randomized trial. Int J Obstet Anesth. 2017;31:68-73. doi: 10.1016/j.ijoa.2017.04.001

9. Hogan QH, Prost R, Kulier A, Taylor ML, Liu S, Mark L. Magnetic resonance imaging of cerebrospinal fluid volume and the influence of body habitus and abdominal pressure. Anesthesiology. 1996;84(6):1341-1349. doi: 10.1097/00000542-199606000-00010

10. Carvalho B, Collins J, Drover DR, Atkinson Ralls L, Riley ET. $\mathrm{ED}(50)$ and $\mathrm{ED}(95)$ of intrathecal bupivacaine in morbidly obese patients undergoing cesarean delivery. Anesthesiology. 2011;114(3):529-535. doi: 10.1097/ALN.0b013e318209a92d

11. Gasim T, Al Jama FE, Rahman MS, Rahman J. Multiple repeat cesarean sections: operative difficulties, maternal complications and outcome. J Reprod Med. 2013;58(7-8):312-318.

12. Lyell DJ. Adhesions and perioperative complications of repeat cesarean delivery. Am J Obstet Gynecol. 2011;205(6

Suppl):S11-S18. doi: 10.1016/j.ajog.2011.09.029

13. Duan G, Yang G, Peng J, Li J, Tang X, Li H. Comparison of postoperative pain between patients who underwent primary and repeated cesarean section: a prospective cohort study. BMC Anesthesiol. 2019;19(1):189. doi: $10.1186 / \mathrm{s} 12871-019-0865-9$

This article meets the Accreditation Council for Graduate Medical Education and the American Board of Medical Specialties Maintenance of Certification competencies for Patient Care, Medical Knowledge, and Practice-Based Learning and Improvement.

(C2021 by the author(s); licensee Ochsner Journal, Ochsner Clinic Foundation, New Orleans, LA. This article is an open (c) (i) access article distributed under the terms and conditions of the Creative Commons Attribution (CC BY) license (creativecommons.org/licenses/by/4.0/legalcode) that permits unrestricted use, distribution, and reproduction in any medium, provided the original author(s) and source are credited. 